Review Article

\title{
Biomechanical Stress in Obturator Prostheses: A Systematic Review of Finite Element Studies
}

\author{
Mohammed A. Mousa $\mathbb{D}^{1,2}$ Johari Yap Abdullah $\mathbb{D}^{3}{ }^{3}$ Nafij B. Jamayet $\left(\mathbb{D},{ }^{4}\right.$ \\ Mohammad Khursheed Alam $\left(\mathbb{D},{ }^{5} \text { and Adam Husein }{ }^{1}\right)^{1}$ \\ ${ }^{1}$ Prosthodontic Unit, School of Dental Sciences, Universiti Sains Malaysia, 16150 Kubang Kerian, Kelantan, Malaysia \\ ${ }^{2}$ Department of Prosthetic Dental Sciences, College of Dentistry, Jouf University, Sakakah, Jouf Province, Saudi Arabia \\ ${ }^{3}$ Craniofacial Imaging Laboratory, School of Dental Sciences, Universiti Sains Malaysia, 16150 Kubang Kerian, Kelantan, Malaysia \\ ${ }^{4}$ Division of Restorative Dentistry, International Medical University, Bukit Jalil, Jalan Jalil Perkasa-19, \\ 57000 Kuala Lumpur, Malaysia \\ ${ }^{5}$ Department of Preventive Dentistry, College of Dentistry, Jouf University, Saudi Arabia
}

Correspondence should be addressed to Mohammed A. Mousa; dr.mohammed.assayed@gmail.com, Mohammad Khursheed Alam; dralam@gmail.com, and Adam Husein; adamkck@usm.my

Received 6 June 2021; Accepted 2 August 2021; Published 17 August 2021

Academic Editor: Du-Hyeong Lee

Copyright (C) 2021 Mohammed A. Mousa et al. This is an open access article distributed under the Creative Commons Attribution License, which permits unrestricted use, distribution, and reproduction in any medium, provided the original work is properly cited.

\begin{abstract}
Aim. This systematic review is aimed at investigating the biomechanical stress that develops in the maxillofacial prostheses (MFP) and supporting structures and methods to optimize it. Design and Methods. A literature survey was conducted for full-text English articles which used FEA to examine the stress developed in conventional and implant-assisted MFPs from January 2010 to December 2020. Results. 87 articles were screened to get an update on the desired information. 74 were excluded based on a complete screening, and finally, 13 articles were recruited for complete reviewing. Discussion. The MFP is subjected to stress, which is reflected in the form of compressive and tensile strengths. The stress is mainly concentrated the resection line and around the apices of roots of teeth next to the defect. Diversity of designs and techniques were introduced to optimize the stress distribution, such as modification of the clasp design, using materials with different mechanical properties for dentures base and retainer, use of dental (DI) and/or zygomatic implants (ZI), and free flap reconstruction before prosthetic rehabilitation. Conclusion. Using ZI in the defective side of the dentulous maxillary defect and defective and nondefective side of the edentulous maxillary defect was found more advantageous, in terms of compression and tensile stress and retention, when compared with DI and free flap reconstruction.
\end{abstract}

\section{Introduction}

Management of patients who presents with such malignancy mostly necessitates surgical removal of a major portion of the palate with ablative surgery. The resultant paltal defect after surgery could be small or massive (when involves removal of a major portion of the palate, maxillary sinus, and/or nasal cavity). The patient's quality of life often collapses following the surgical resection of the tumor mass because of the corruption of function, speech, and aesthetics [1]. To overcomes the functional and psychological impact of the surgery, a surgical microvascular and/or prosthetic reconstruction must be carried out to improve the patient quality of life $[2,3]$. Surgical rehabilitation of maxillary defects is not always possible due to the lack of donor sites, size of the defect, general health of the patient, and the risk of morbidity [4]. Maxillofacial prostheses (MFPs) are considered a cost-effective treatment option to reconstruct the lost dentition and missing structures in patients suffering from major maxillary defects [5, 6]. Even though many classifications have been introduced to distinguish the maxillary defect [7-10]; Aramany's classification was the most one followed by researchers due to its simplicity and smoothly communication among the maxillofacial prosthodontists [11]. 
When removable MFPs are used for rehabilitation of cases with major maxillary defects, the prostheses and their supporting structures are subjected to enormous pressure depending on the size and location of the defect, lack of adequate bone support, weight of the prostheses, the poor flexural strength, and low fatigue resistance of the prosthetic framework [12]. The developed pressure results in a concentration of the stress on the remaining part of supporting structures leading to bone resorption around the abutments and, eventually, failure of the prosthesis. Implant-assisted MFPs show many advantages comparing to the conventional ones, such as preservation of the remaining supporting structures, improvement of retention and stability, improvement of chewing efficiency, and improvement of patient's quality of life [13]. There is a direct relationship between success in dental treatment and biomechanics of materials used in dentistry [14]. The study of stress in prosthetic restorations has been reported before using different methods such as strain-gauge measurement, photoelastic stress analysis, and statistical finite element analysis (FEA) $[15,16]$. FEA provides noninvasive reproducible qualitative and quantitative 2D and 3D information of biomechanical characteristics of dental prostheses and supporting structures with no need for ethical considerations when compared to other experimental methods [17-19]. FEA is carried out in three stages; the first stage is referred to as the "preprocessing stage" and it entails the creation of the FE model as well as specifying the properties of the materials. FE model can be generated in $3 \mathrm{D}$ by exporting the data from cone beam computerized tomography (CBCT) or magnetic resonance imaging (MRI), in (.stl) file format. Following the generation of FE model, the properties of the materials (and tissues) can be specified $[20,21]$. The second stage is called "loading and boundary conditions" and it involves identifying the areas chosen to be the constrain and the area chosen to receive the load, and then the required load can be applied to the area of interest. The "postprocessing stage" which involves data analysis and result interpretation, is the third stage of FEA. Evaluation of biomechanical stress develps in different scenarios of MFPs has been done in literature, however, a systematic review of stress develops in the different scenarios MFPs, up to the authors' knowledge, has not yet been received in the dental literature. This was the purpose of this systematic review; to appraise the studies that used FEA to evaluate the biomechanical stress developed in removable MFPs and their supporting structures.

\section{Study Design and Methods}

This study was done after getting approval from the Human Research Ethics Committee of Universiti Sains Malaysia (HREC/USM) with JEPeM Code: USM/JEPeM/21030222 (Ph.D. proposal). The study followed the Preferred Reporting Items for Systematic Reviews and Meta-Analyses (PRISMA) guidlines. The questions of the research were formulated with the aid of the PICO format; while $(\mathrm{P})$ is for the participants, (I) for the intervention, (C) for the comparison, and (O) for the outcome [22]. In scenarios with different forms of maxillary defects $(\mathrm{P})$, what are the influences of maxillofa- cial prostheses (I) that fabricated with different designs and materials $(\mathrm{C})$ on the distribution of stress and displacement of the prostheses during function $(\mathrm{O})$ ? An electronic search was conducted using the PubMed, Scopus, and Web of Science databases research tools. The inclusion criteria, as shown in Table 1, were the FEA studies, those conducted in English from January 2010 to December 2020, and only the studies that evaluated the stress on maxillary obturator prostheses. The exclusion criteria, as shown in Table 1, were in vivo studies, in vitro experimental studies, literature reviews, the articles that used FEA to estimate the stress in conventional fixed, removable partial, and complete denture, the articles that used methods other than FEA, letters to the editor, and unpublished data. The research was done by two authors (M.A.M and J.Y.A) independently conducted an electronic search on the 3 identified databases (PubMed, Scopus and Web of Science). The authors used the keywords "finite element analysis" and "obturators" for the preliminary survey. The articles were primarily screened by their title and abstract then by assessing the full text. In the selected articles, further research was performed in their references and citations for the possibility of including more articles. The quality assessment of the selected studies was not applicable as it is a mathematical way of estimating stress. The search was done to find answers to two questions. The first question was "where the stress distributed in the MFPs and supporting structures?", while the second question was "What are the factors affecting the biomechanical stress distribution in the MFPs?"

\section{Results}

During the nominated time of the study, 87 articles primarily were included in the survey. Out of these articles, 70 were excluded (based on initial screening of their titles and abstracts). Reasons for exclusion were either studies that were not related to the objectives of the current review, studies with duplicating results, or studies written in languages other than English. Four more studies were excluded after reading their methodology [23-26]. One of them evaluated the stress in congenitally unilateral palatal cleft scenario [25], one used mainly in vivo approach with no interpretation in the result and discussion sections regarding FEA [23], and four different studies were duplicated in their methodology and results $[24,26]$, so we chose the earlier studies $[13,27]$. Finally, 13 articles were recruited for this review [13, 27-38]. The number of primary surveyed articles, number of excluded articles, reasons for exclusion, and the final recruited articles are shown in Figure 1.

Table 2 shows the summary of the studies that delivered on dentate maxillary defect scenarios, showing the type of research, the type of the maxillary defect examined, the design of the prostheses, the magnitude of the applied load, and assessment of the stress distribution in supporting tissues and the overlying prosthesis.

Table 3 shows the studies conducted to evaluate the stress developed in edentulous maxillary defects and their supporting structures. The results and findings were collected to identify the biomechanical stress developed in the maxillofacial 
TABLE 1: The inclusion and exclusion criteria of the present study.

Inclusion criteria

(1) The studies conducted between January 2010 and December 2020

(2) Studies conducted in the English language

(3) In vitro mathematical studies

(4) Studies conducted only on partial and complete removable maxillofacial obturators

(5) The case with surgical maxillary defect

(6) Studies used only FE methods to predict the distribution of the stress and displacement in MFP
Exclusion criteria

(1) The studies conducted out of the inclusion time range

(2) Studies conducted in a language other than English

(3) In vivo and in vitro lab experimental studies

(4) Studies conducted on conventional partial and complete removable dentures

(5) Studies conducted on fixed prostheses

(6) The scenarios with congenital maxillary defect

(7) Studies conducted on partial MFP with complete acrylic base

(8) Studies that used other than FE method to predict the stress

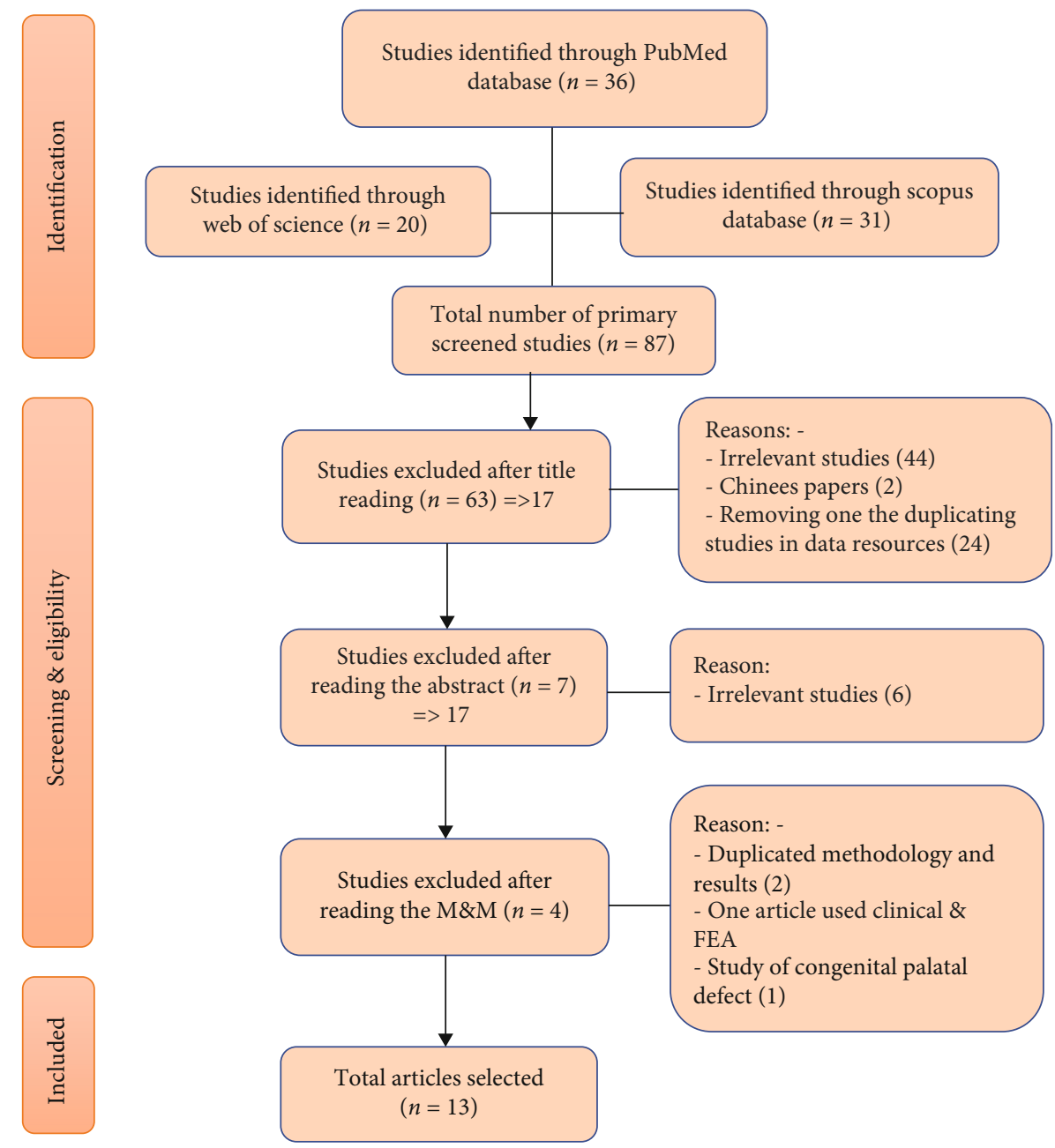

Figure 1: Flow chart for search process indicating numbers $(n)$ of included and excluded studies.

prostheses and their supporting structures and how to manage it.

Except for three studies that followed Okay's classification $[25,31,38]$, most of the recruited studies adhered to Aramany's classification $[25,31,38]$. Having read the methodology of the studies that used Okay's classification, we applied Aramany's equivalent design to their scenarios, to facilitate the comparison among the studies. Table 4 shows the studies that examined the different scenarios of Aramany classification and the scenarios which failed to recieve attention in the literature.

The reviewed studies were aimed at identifying the maximum (tensile force) and minimum (compressive force) principle stress in the examined designs except for one study 


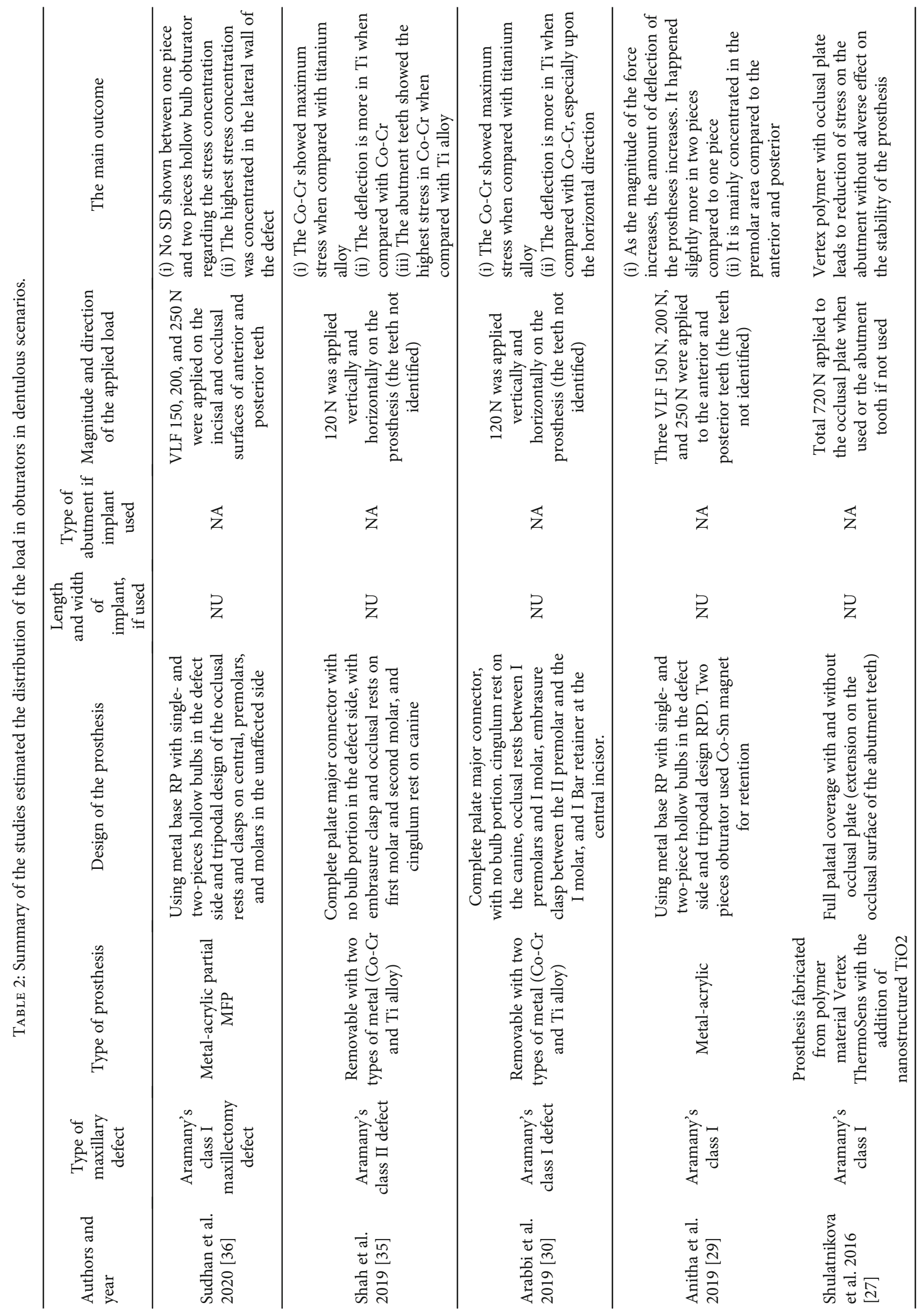




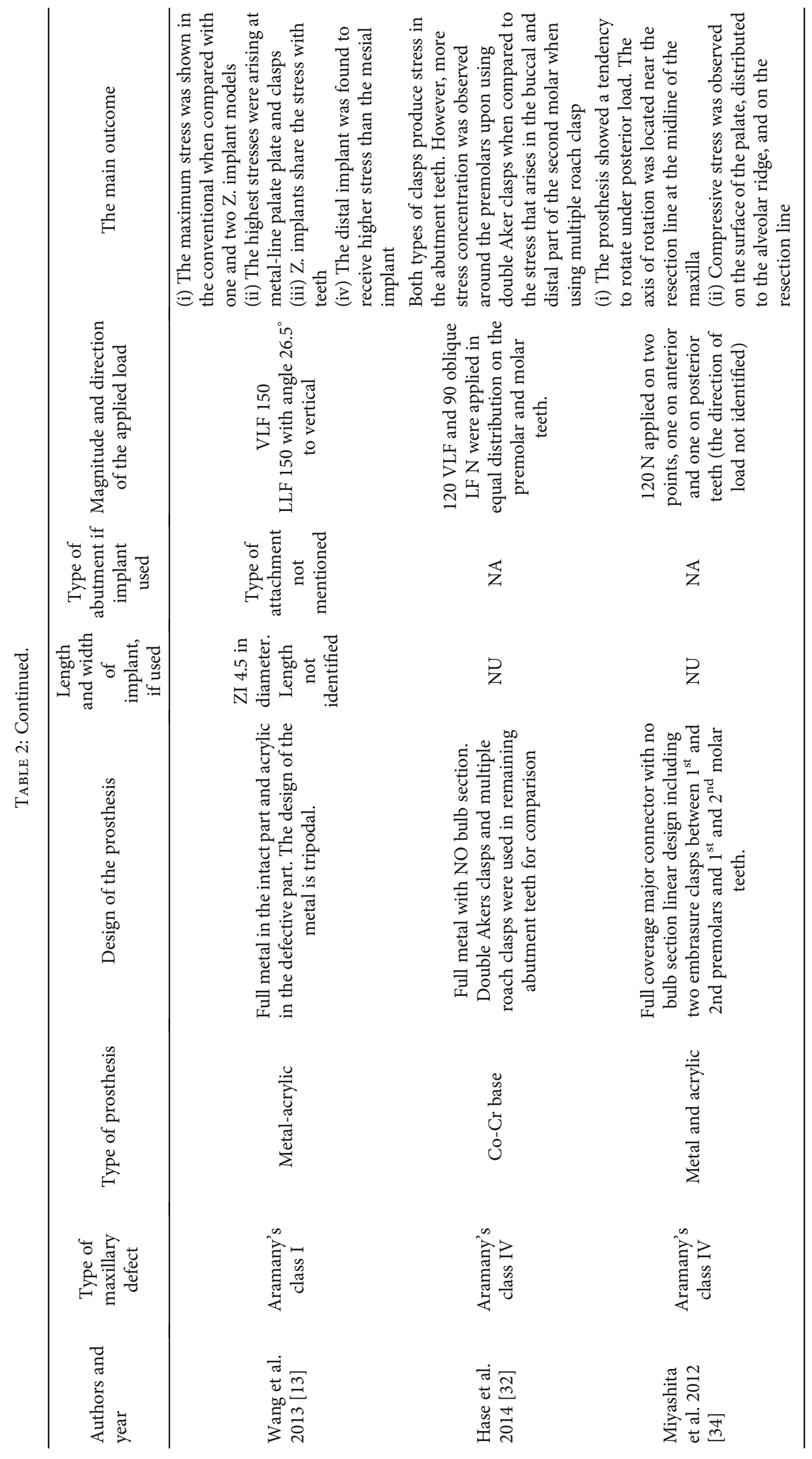




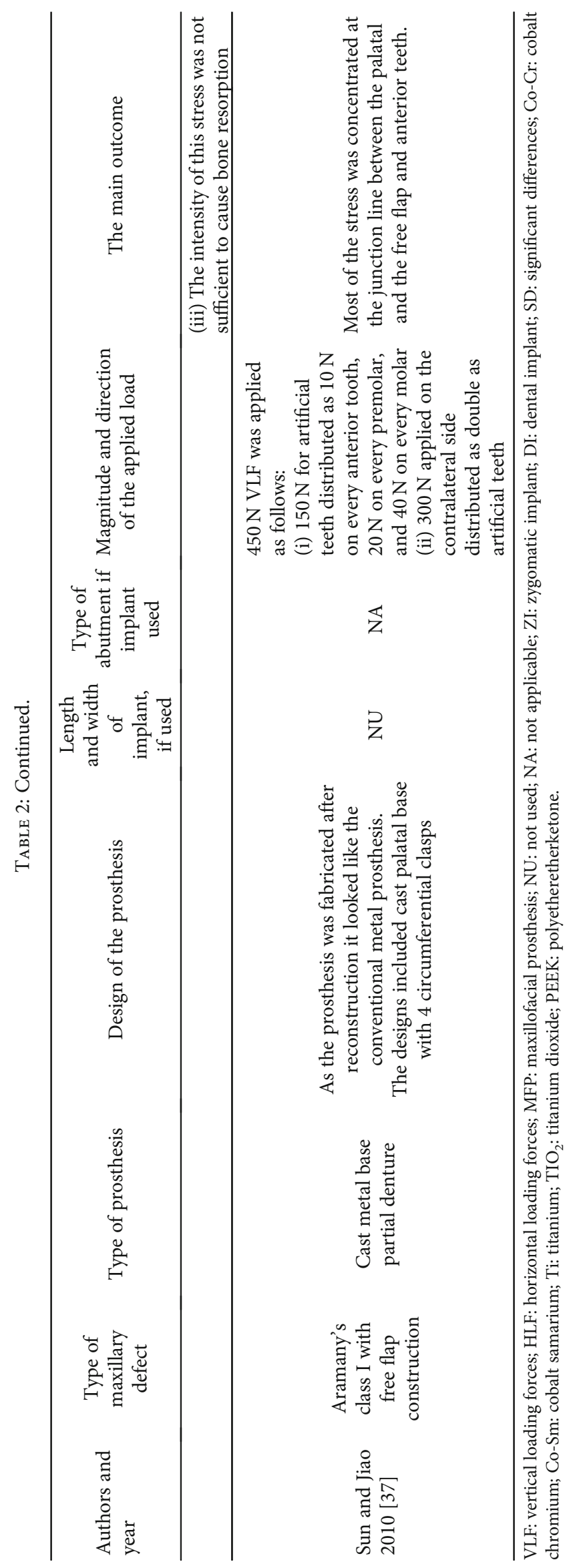




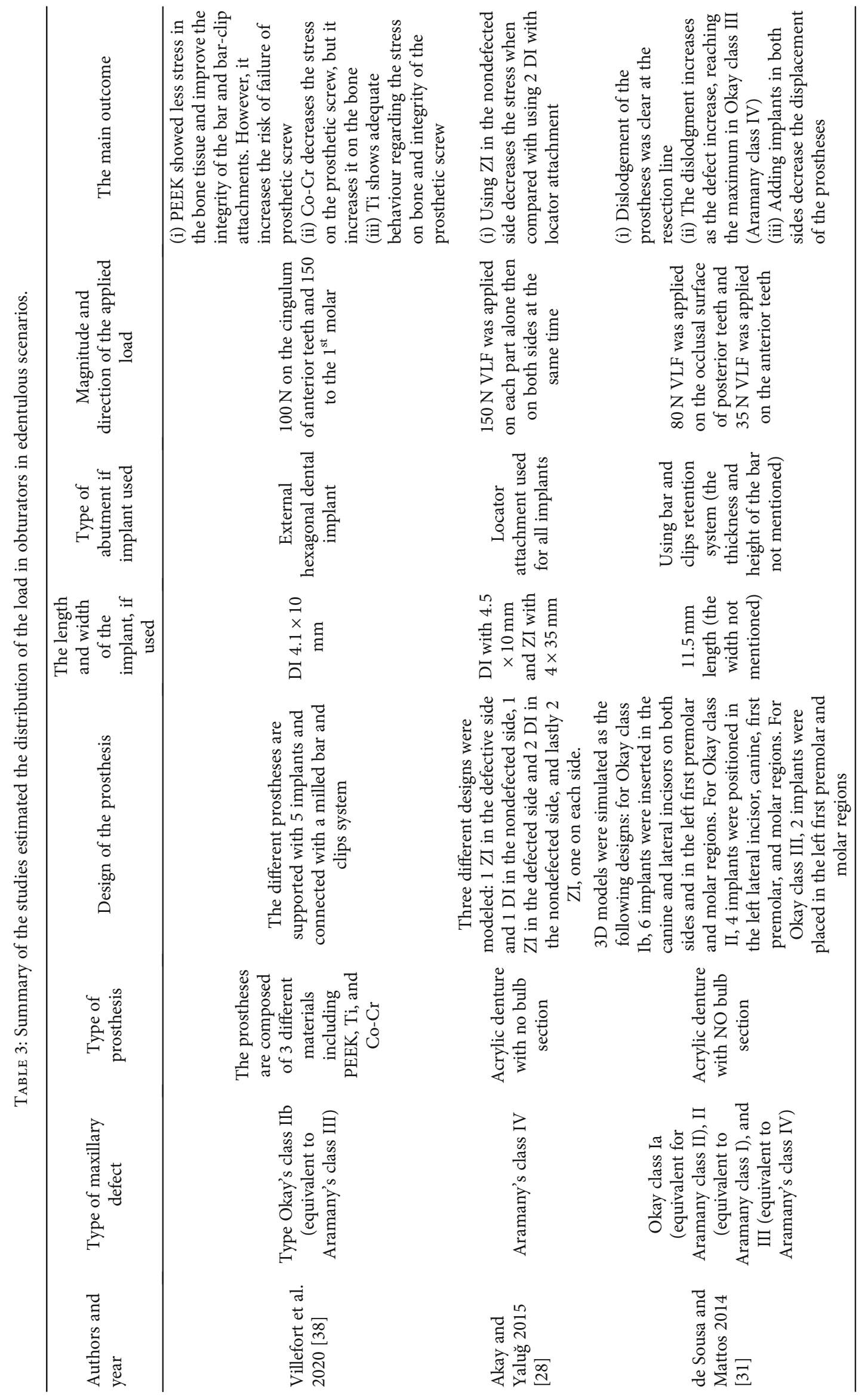




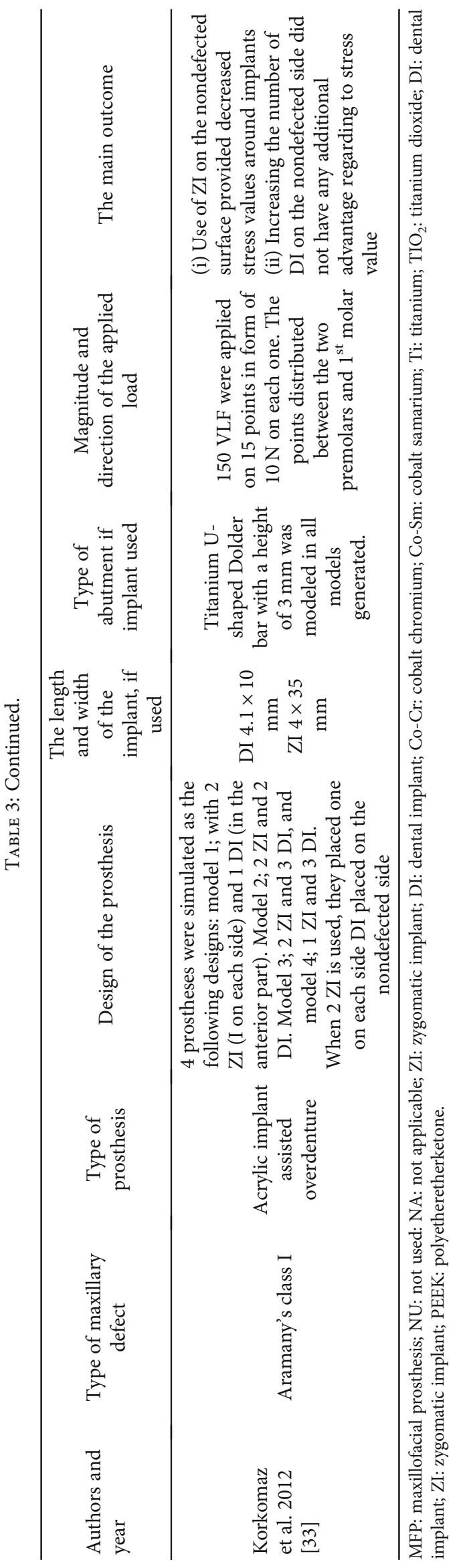


TABLE 4: Summary of the selection process of the review.

\begin{tabular}{|c|c|c|c|c|}
\hline & & & & Aims \\
\hline $\begin{array}{l}\text { maxillary } \\
\text { defect }\end{array}$ & scenario & The study & Stress & Displacement \\
\hline Aramany's & Dentulous & $\begin{array}{l}\text { Sudhan et al. } 2020 \text { [36], Arabbi et al. } 2019 \text { [30], Anitha et al. } 2019 \text { [29], Shulatnikova } \\
\text { et al. } 2016 \text { [27], Wang et al. } 2013 \text { [13], and Sun and Jiao } 2010 \text { [37] }\end{array}$ & $\sqrt{ }$ & $\sqrt{ }$ \\
\hline & Edentulous & de Sousa and Mattos 2014 [31] and Korkmaz et al. 2012 [33] & $\sqrt{ }$ & $\sqrt{ }$ \\
\hline Aramany's & Dentulous & Shah et al. 2019 [35] & $\sqrt{ }$ & $\sqrt{ }$ \\
\hline class II & Edentulous & de Sousa and Mattos 2014 [31] & $\sqrt{ }$ & $\sqrt{ }$ \\
\hline Aramany's & Dentulous & - & - & - \\
\hline class III & Edentulous & Villefort et al. 2020 [38] and de Sousa and Mattos et al. 2014 [31] & $\sqrt{ }$ & $\sqrt{ }$ \\
\hline Aramany's & Dentulous & Hase et al. 2014 [32] and Miyashita et al. 2012 [34] & $\sqrt{ }$ & $\sqrt{ }$ \\
\hline class IV & Edentulous & Akay and Yaluğ 2015 [28] and de Sousa and Mattos 2014 [31] & $\sqrt{ }$ & $\sqrt{ }$ \\
\hline Aramany's & Dentulous & - & - & - \\
\hline class V & Edentulous & - & - & - \\
\hline Aramany's & Dentulous & - & - & - \\
\hline class VI & Edentulous & - & - & - \\
\hline
\end{tabular}

that assessed the differences in displacement between singleand two-piece closed hollow-bulb obturators [29]. In the reviewed studies, the stress was estimated in the prostheses for partially edentulous scenarios $[13,27,29,30,32,34-$ 37], while in another it was examined in completely edentulous scenarios [25, 28, 31, 33, 38]. Six of the 13 studies reviewed in the current review, examined the influences of implant/s on the distribution of stress in the prostheses. Out of these six studies, only one was conducted in a dentate scenario [13], while the other five were for edentulous scenarios $[25,28,31,33,38]$. The used length and width of DI in all studies were the standard $(4.1-4.5 \times 10 \mathrm{~mm})[28,31,33$, 38], while ZI was $4 \times 35 \mathrm{~mm}$ in all scenarios [13, 28, 33]. The stress distribution of single-piece hollow bulb section was examined by two studies [13, 25], while the two-piece was examined by two other studies as well $[29,36]$, and this mostly was to simulate the real situation.

There was vast differences in the magnitude of applied loads among the studies. The main reason for this is owing to the variety of the occlusal forces that can be found in the population, which are dependent on gender, age, general health, natural dentition, and anterior or posterior teeth. However, the mean maximum force 120-150 N were selected, either individually or collectively, in most of the reviewed studies, as it considers the mean maximum force for patients with remaining natural teeth and wearing removable obturator prostheses [13, 29, 30, 32, 35, 36].

\section{Discussion}

The purpose of this review was to compile all current information on the stress distribution developed in MFP and supporting structures, together with and the factors affecting it, from all FEA studies published within the last decade.

In the past decade, FEA has gained acceptance as a noninvasive and reliable method for simulating different dental defects and their corresponding prostheses, as well as analyz- ing the distribution of the stress within these prostheses and supporting structures [26]. However, the application of FEA has been used in limits in the identification of stress distribution in MFPs which may be due to the complexity of modeling the defects, simulation of the corresponding prosthesis, and the time involved.

Aramany's classification was the dominant classification that has been followed by most of the researchers. Essentially, this is mainly because of the simplicity of design and wide coverage of Aramany's classification which can be observed when applied to the other different classifications. Despite this, Aramany's classification overlooked the vertical extension of the maxillary defect, and thus, those authors who followed Aramany classification mistakenly assumed it does not exists [2].

There is a dependence between the stress developed in MFPs and magnitude, location, direction of the applied load, and number of remaining dentitions as well. As the load applied to the prosthesis does increase, the stress concentration in the prostheses and associated structures increases. This stress is shown as a compressive force, which concentrates at the resection line $[31,34]$. Moeover, the stress developed within the prosthesis is increased as the number of remaining teeth decreases and as the size of the defect increases $[32,34]$. The stress can be developed in either tensile or compressive form depending on where the load is applied. When the load is applied to the posterior portion of the prosthesis, the stress mainly developed in a compressive form and observed on the anterior midline. When the load applied on the anterior part of the prosthesis, the main stress becomes in the form of tensile and observed throughout the midline region [34].

In the studies that evaluated dentulous scenarios of Aramany's class I and class II palatal defect, they found that the maximum stress concentration is located around the cervical half of the roots of the teeth next to the resection, central, and lateral incisors of the contralateral side in class I or canine in 
class II [13, 30, 35]. The cobalt-chromium alloy was found to produce more stress on the remaining teeth, when used as a major connector and retainers, compared to titanium alloy which showed more flexibility. Desbite this, due to titanium decreased rigidity, the major connector is deflected toward the areas where the force is applied [30,35]. The use of Vertex polymer as a retainer with an occlusal plate on the main abutment teeth was found to reduce stress on the teeth without compromising the stability of the prosthesis [24]. There were no significant differences in the distribution of stress between the single- and two-piece hollow obturators, although the two-piece obturator showed a slight lower stress value than the single-piece obturator $[29,36]$.

Free flap reconstruction of unilateral dentate maxillary defect is used, as a surgical method, to simplify the prosthetic rehabilitation. The greates stress concentration, however, was found at the junction of the flap and the palatal bone under obturator. This stress was found four times more than the stress developed under traditional obturator, thus results in rapid loss of bone support at the junction between the flap and the palate, causing instability of the obturator [37]. Another way to decrease the stress concentration in a dentate Aramany's class I was to assist and retain the MFP with ZI/s, since the DI cannot be used in the defective side. When one or two ZI are added to assist the MFP, the ZI shares the stress with the abutment teeth on the contralateral side, thereby reducing the torque on the abutment teeth and reducing the rotation of the prosthesis toward the defect [13].

The design of MFPs in dentate scenarios receiving the least attention in literature is Aramany's class III. To the best of authors' knowledge, there was a lack in literature, up to the authors' knowledge, of this design from the perspective of FEA. This may be because Aramany's class III might resemble, to an extensive degree, the conventional Kennedy class III. In contrast, Aramany's class I and IV were the two designs which garnered the most attention $[13,27,29,30$, $32,36]$. Owing to the extreme defect associated with these scenarios, which have a substantial impact on the biomechanical stress in the corresponding prostheses and their supporting structures, might prompted the researchers to investigate them. The massive bone defect makes the MFPs tend to rotate toward the defect around the midline of the remaining part of the maxilla, which affects the stability and periodontium of the remaining abutment teeth [34]. It was possible to reduce the stress in Aramany's class IV by modifying the clasp design [32]. Researchers found that the multiple roach clasps reduce the stress on the MFP supporting structure when compared with multiple Aker's clasps [32]. There was a lack of literature, up to our knowledge, about the influences of assisting the MFP in Aramany's class IV with ZI.

In contrast to the lack of coverage to some of Aramany's classification defects in dentate scenarios, there has been wide literature coverage of the various types of edentulous Aramany classification over the last decade [28, 31, 33, 38]. In the study done by de Sousa and Mattos, they follow Okay's classification Ia, II, and III maxillary defects, which are equivalent to Aramany's class II, I, and IV, respectively [31]. Same as in dentate maxillary defects, the displacement of the MFP at the resection line increases as the defect increases, reaching a maximum in Okay's class III (Aramany's class IV) [31]. Even though adding DI on the nondefective side was found to decrease the stress on the remainder of supporting structure, the implant/s are still subjected to high compressive stress especially when the defect becomes massive as in Okay's class III situations. The stress in Okay's class III is tensile in its dominant form and usually concentrated around the cervical part of the cortical bone around DI, which may jeopardize the survival of the implants. One method to decrease the stress on the DI on the nondefective side is adding one (or two) ZI on the defective side [28], or adding one ZI on each side, which was found beneficial in reducing the stress comparing adding two or three DI in the nondefective side [33].

The amount of stress concentration depends not only on the type and number of the implants but also on the type of prosthetic materials, the type of the connectors, and the abutments of implants. Although the polyetheretherketone (PEEK) showed the least stress on the residual ridge (bone), it showed the highest stress concentration in the prosthetic screws and clips. In contrast to PEEK, Co-Cr showed the highest stress on the bone but the lowest stress on the prosthetic screws. The supporting bone and prosthetic screws showed adequate stress concentration with titanium framework [38].

There is no disputation, that forces of occlusal and masticatory function are completely transmitted to restoration and supporting structure without loss $[39,40]$. As per the reviewed studies, these forces could be concentrated in certain portions in the prostheses and their supporting structure, leading to permanent deformation (or even fracture to these portions) and/or tissue resorption to the supporting structure. From the authors' point of view, the key factor for the success or failure of MFPs is to evaluate the pattern in which stresses are transferred to the supporting structures to minimize it.

This systematic review showed the deficiency in the literature regarding different designs of the different scenarios of maxillary defects and their corresponding prostheses. Further researches are needed to examine the overlooked scenarios of maxillary defects as shown in this review.

This review focused only on the FEA studies to identify the stress concentration in MFPs and the methods to reduce this stress. Despite its noninvasive and flexible nature, FEA has many inherited limitations when comes to simulating maxillary defect and their corresponding prostheses. Some, but not all, of these limitations include the homogeneity of the used materials (ignoring the manufactures and laboratory errors during processing), isotropic linearity (assuming the material when respond to load, shows the same reaction in all direction), biology of the tissues (assuming the bone showing the same density in all areas), osseointegration (assuming 100\% osseointegration), and the other physiologic differences that exist among the patients. Clinical validation using methods, such as photoelastic stress analysis and strain gauge analysis, would be crucial to validate the FEA.

\section{Conclusion}

Within the limitation of this review, we can conclude the following 
(1) The stress and displacement of MFPs are highly influenced by the sizes and characters of maxillary defects, availability of adequate undercuts, and health and position of the remaining dentitions

(2) The stress is mainly concentrated on the resection side and the apices of the teeth next to the defect

(3) Using DI in the nondefective of maxillary defect reduces the stress on the supporting structure as the implant share the stress with the abutments

(4) Adding ZI in the defective side of dentulous and in the defective and nondefective sides in edentulous maxillary defect is considered a key factor in reducing the displacement of maxillofacial prostheses. It may also decrease the need to DI, the need to use clasps on the teeth next to the resection, and - to massive surgical free flap reconstruction

\section{Data Availability}

All data are available within the manuscript.

\section{Conflicts of Interest}

The authors declare that they have no conflicts of interest.

\section{References}

[1] V. Vander Poorten, J. Meulemans, and P. Delaere, "Midface prosthetic rehabilitation," Current Opinion in Otolaryngology \& Head and Neck Surgery, vol. 24, no. 2, pp. 98-109, 2016.

[2] R. Ali, A. Altaie, and B. Nattress, "Rehabilitation of oncology patients with hard palate defects part 1: the surgical planning phase," Dental Update, vol. 42, no. 4, pp. 326-335, 2015.

[3] T. H. Farook, M. A. Mousa, and N. B. Jamayet, "Method to control tongue position and open source image segmentation for cone- beam computed tomography of patients with large palatal defect to facilitate digital obturator design," Journal of Oral and Maxillofacial Surgery, Medicine, and Pathology, vol. 32, no. 1, pp. 61-64, 2020.

[4] M. Sakuraba, Y. Kimata, Y. Ota et al., "Simple maxillary reconstruction using free tissue transfer and prostheses," Plastic and Reconstructive Surgery, vol. 111, no. 2, pp. 594-598, 2003.

[5] B. Shrestha, B. B. Basnet, and G. Adhikari, "A questionnaire study on the impact on oral health-related quality of life by conventional rehabilitation of edentulous patient," BDJ Open, vol. 6, no. 1, p. 3, 2020.

[6] I. I. Artopoulou, E. C. Karademas, N. Papadogeorgakis, I. Papathanasiou, and G. Polyzois, "Effects of sociodemographic, treatment variables, and medical characteristics on quality of life of patients with maxillectomy restored with obturator prostheses," The Journal of prosthetic dentistry, vol. 118, no. 6, pp. 783-789.e4, 2017.

[7] D. J. Okay, E. Genden, D. Buchbinder, and M. Urken, "Prosthodontic guidelines for surgical reconstruction of the maxilla: a classification system of defects," The Journal of Prosthetic Dentistry, vol. 86, no. 4, pp. 352-363, 2001.

[8] M. A. Aramany, "Basic principles of obturator design for partially edentulous patients. Part II: design principles," Journal of Prosthetic Dentistry, vol. 86, no. 6, pp. 562-568, 2001.
[9] J. S. Brown and R. J. Shaw, "Reconstruction of the maxilla and midface: introducing a new classification," The Lancet Oncology, vol. 11, no. 10, pp. 1001-1008, 2010.

[10] N. D. Futran and E. Mendez, "Developments in reconstruction of midface and maxilla," The Lancet Oncology, vol. 7, no. 3, pp. 249-258, 2006.

[11] F. Keyf, "Obturator prostheses for hemimaxillectomy patients," Journal of Oral Rehabilitation, vol. 28, no. 9, pp. 821-829, 2001.

[12] P. Pfeiffer, C. Rolleke, and L. Sherif, "Flexural strength and moduli of hypoallergenic denture base materials," The Journal of Prosthetic Dentistry, vol. 93, no. 4, pp. 372-377, 2005.

[13] M. Wang, X. Qu, M. Cao, D. Wang, and C. Zhang, "Biomechanical three-dimensional finite element analysis of prostheses retained with/without zygoma implants in maxillectomy patients," Journal of Biomechanics, vol. 46, no. 6, pp. 11551161, 2013.

[14] W. G. Assunção, V. A. Ricardo Barão, L. F. Tabata, E. A. Gomes, J. A. Delben, and P. H. dos Santos, "Biomechanics studies in dentistry," Journal of Craniofacial Surgery, vol. 20, no. 4, pp. 1173-1177, 2009.

[15] A. A. Pesqueira, M. C. Goiato, H. G. Filho et al., "Use of stress analysis methods to evaluate the biomechanics of oral rehabilitation with implants," Journal of Oral Implantology, vol. 40, no. 2, pp. 217-228, 2014.

[16] J. P. M. Tribst, A. M. d. O. Dal Piva, and A. L. S. Borges, "Biomechanical tools to study dental implants: a literature review," Brazilian Dental Science, vol. 19, no. 4, pp. 5-11, 2016.

[17] M.-L. Hsu and C.-L. Chang, "Application of finite element analysis in dentistry," in Finite Element Analysis, pp. 44-60, IntechOpen Limited, London, 2010.

[18] M. A. Mousa, N. Jamayet, E. Lynch, and A. Husein, "Biomechanical stress in removable complete dental prostheses: a narrative review of finite element studies," Journal of International Oral Health, vol. 12, p. 413, 2020.

[19] M. Mousa, J. Abdullah, and N. Jamayet, "Biomechanics in removable partial dentures: a literature review of FEA based studies," BioMed Research International, vol. 5699962, 2021.

[20] C.-C. Ko, E. P. Rocha, and M. Larson, "Past, present and future of finite element analysis in dentistry," in Finite Element Analysis - From Biomedical Applications to Industrial Developments, pp. 1-25, IntechOpen Limited, London, 2012.

[21] P. Magne, "Efficient 3D finite element analysis of dental restorative procedures using micro-CT data," Dental Materials, vol. 23, no. 5, pp. 539-548, 2007.

[22] C. Schardt, M. B. Adams, T. Owens, S. Keitz, and P. Fontelo, "Utilization of the PICO framework to improve searching PubMed for clinical questions," BMC Medical Informatics and Decision Making, vol. 7, no. 1, 2007.

[23] X. Z. Qu, M. Y. Wang, H. S. Ong, and C. P. Zhang, "Postoperative hemimaxillectomy rehabilitation using prostheses supported by zygoma implants and remaining natural teeth," Clinics, vol. 71, no. 10, pp. 575-579, 2016.

[24] O. A. Shulyatnikova, G. I. Rogozhnikov, L. E. Leonova, V. A. Lokhov, A. F. Shulyatyev, and L. A. Mozgovaya, "Biomechanical analysis clamping fixation system for prosthesis-obturator," Russian Journal of Biomechanics, vol. 21, pp. 199-204, 2017.

[25] G. SoğAnci and H. YazicioğLu, "Evaluation of stress distribution of mini dental implant-supported overdentures in complete cleft palate models: a three-dimensional finite element 
analysis study," Cleft Palate-Craniofacial Journal, vol. 53, no. 1, pp. 73-83, 2016.

[26] G. Wang, S. Zhang, C. Bian, and H. Kong, "Verification of finite element analysis of fixed partial denture with in vitro electronic strain measurement," Journal of Prosthodontic Research, vol. 60, no. 1, pp. 29-35, 2016.

[27] O. A. Shulatnikova, G. I. Rogozhnikov, V. A. Lokhov, and A. F. Shulatyev, "Biomechanical analysis of post-resection prosthesis-obturator made from polyamide reinforced by nanostructured titanium dioxide," Russian Journal of Biomechanics, vol. 20, pp. 326-332, 2016.

[28] C. Akay and S. Yaluğ, "Biomechanical 3-dimensional finite element analysis of obturator protheses retained with zygomatic and dental implants in maxillary defects," Medical science monitor: international medical journal of experimental and clinical research, vol. 21, p. 604, 2015.

[29] K. Anitha, N. Gopi Chander, V. Karthikeyan, and R. Hariharasudan, "Finite element analysis of displacement with single and two piece hollow bulb obturator prosthesis," medical journal armed forces india, vol. 75, no. 4, pp. 395399, 2019.

[30] K. C. Arabbi, T. D. Shah, M. Sharanappa, and S. K. Subbaiah, "A three-dimensional finite element analysis of Aramany class I obturator fabricated with different alloys," Journal of Pharmacy \& Bioallied Sciences, vol. 11, p. S24, 2019.

[31] A. A. de Sousa and B. S. C. Mattos, "Finite element analysis of stability and functional stress with implant- supported maxillary obturator prostheses," The Journal of Prosthetic Dentistry, vol. 112, no. 6, pp. 1578-1584, 2014.

[32] H. Hase, A. Shinya, D. Yokoyama, A. Shinya, and Y. Takahashi, "Three-dimensional finite element analysis of Aramany Class IV obturator prosthesis with different clasp designs," Dental Materials Journal, vol. 33, no. 3, pp. 383388, 2014.

[33] F. M. Korkmaz, Y. T. Korkmaz, S. Yaluğ, and T. Korkmaz, "Impact of dental and zygomatic implants on stress distribution in maxillary defects: a 3-dimensional finite element analysis study," Journal of Oral Implantology, vol. 38, no. 5, pp. 557-567, 2012.

[34] E. R. Miyashita, B. S. C. Mattos, P. Y. Noritomi, and H. Navarro, "Finite element analysis of maxillary bone stress caused by Aramany Class IV obturator prostheses," The Journal of Prosthetic Dentistry, vol. 107, no. 5, pp. 336-342, 2012.

[35] T. D. Shah, Y. Naveen, P. Kattimani, G. Kamath, K. Shah, and A. Raza, "Stress distribution \& deflection in an Aramany class II obturator fabricated with cobalt-chromium \& titanium alloys - 3D FEA," The Saudi dental journal, vol. 31, no. 2, pp. 251-257, 2019.

[36] R. H. Sudhan, G. N. Chander, and K. V. Anitha, "Finite element stress analysis of Aramany class I maxillectomy defect with single-and two-piece closed bulb obturators," in Gerodontology, Springer Nature in partnership with British Dental Association, [London], 2020.

[37] J. Sun, “Three-dimensional finite element analysis of the prosthetic rehabilitation for unilateral maxillary defect after free flap reconstruction," in 2010 3rd International Conference on Biomedical Engineering and Informatics, Yantai, China, 2010.

[38] R. F. Villefort, J. P. M. Tribst, A. M. D. O. Dal Piva et al., "Stress distribution on different bar materials in implant-retained palatal obturator," PLoS One, vol. 15, no. 10, p. e0241589, 2020.
[39] A. Merdji, B. Bachir Bouiadjra, B. Ould Chikh et al., "Stress distribution in dental prosthesis under an occlusal combined dynamic loading," Materials \& Design (1980-2015), vol. 36, pp. 705-713, 2012.

[40] Y. Matsudate, N. Yoda, M. Nanba, T. Ogawa, and K. Sasaki, "Load distribution on abutment tooth, implant and residual ridge with distal- extension implant-supported removable partial denture," Journal of Prosthodontic Research, vol. 60, no. 4, pp. 282-288, 2016. 